\title{
EXPECTED PHYSICS PERFORMANCE OF THE LHCB EXPERIMENT
}

\author{
OLIVIER LEROY \\ Centre de Physique des Particules de Marseille \\ 163 avenue de Luminy Case 902 \\ 13288 Marseille cedex 9, France \\ E-mail: Olivier.Leroy@in2p3.fr
}

\begin{abstract}
$\mathrm{LHCb}$ is a dedicated B-physics experiment at the LHC. It is currently under construction, to be ready for first collisions in 2007. We give an overview of the expected physics performance: sensitivity in typical channels for study of $\mathrm{B}_{\mathrm{s}}^{0}$ mixing, $\mathrm{CP}$ violation and rare $\mathrm{B}$ decays.
\end{abstract}

\section{Introduction}

The LHCb experiment, its tracking and particle identification systems have been introduced elsewhere in this conference ${ }^{1,2}$. Here we will illustrate the $\mathrm{LHCb}$ physics performance with some key measurements. More detailed results can be found in ${ }^{3}$.

$\mathrm{CP}$ violation is described in the Standard Model by a complex phase in the CKM matrix ${ }^{4,5}$. The $\mathrm{B}$ meson sector is a place where theoretical predictions can be precisely compared with experimental results. LHCb will measure precisely fundamental parameters of the Standard Model like the phases $\arg V_{\mathrm{td}}=-\beta, \arg V_{\mathrm{ub}}=-\gamma$ and $\arg V_{\mathrm{ts}}=-\phi_{s} / 2+\pi$. We

will mesasure the $\mathrm{B}_{\mathrm{s}}^{0}$ oscillation frequency and explore many rare decays involving radiative, electroweak and gluonic penguin amplitudes. There is also a $\mathrm{B}_{\mathrm{c}}^{+}$and b-baryon physics program and our trigger is designed to face unexpected decays. With all these measurements, we will over constrain the unitarity triangles in order to search for inconsistencies and to exhibit new physics. Indeed new physics scenarii propose new particles and new couplings which can modify the amplitudes of penguin and box diagrams.

Physics potential of LHCb is estimated using Monte Carlo simulation. Proton-proton collisions are generated using PYTHIA 6.2 including hard QCD processes, single and double diffraction. Multiple parton interactions are tuned to reproduce the track multiplicities observed at SPS and Teva- 
tron energies. The particles are propagated through the detector using Geant simulation. Each sub-dector response, resolution, efficiency, noise and cross-talk is taken into account using test beam data. Then the offline reconstruction is done using a full pattern recognition program, including track finding and $\mathrm{RICH}$ reconstruction. More than $40 \mathrm{M}$ b $\overline{\mathrm{b}}$ events have been generated to perform detailed background studies.

The $\mathrm{B}_{\mathrm{s}}^{0}$ proper time resolution ${ }^{1}$ is around $43 \mathrm{fs}$. The flavour of the signal $\mathrm{B}$ meson is tagged using fragmentation kaon or pion as well as the opposite b-hadron decay products: kaon, leptons and vertex charge. All information is combined using a neural network. Typical effective tagging efficiencies vary between $4-5 \%$ for $\mathrm{B}^{0}$ and $7-9 \%$ for $\mathrm{B}_{\mathrm{s}}^{0}$.

\section{2. $\mathrm{B}_{\mathrm{s}}^{0}$ mixing, phase and $\Delta \Gamma_{s}$}

The $\mathrm{B}_{\mathrm{s}}^{0}$ oscillation frequency, $\Delta m_{\mathrm{s}}$ will be measured using $\mathrm{B}_{\mathrm{s}}^{0} \rightarrow \mathrm{D}_{\mathrm{s}}^{-} \pi^{+}$ decays. The statistical uncertainty will be $0.01 \mathrm{ps}^{-1}$, with $2 \mathrm{fb}^{-1 \mathrm{a}}$ if $\Delta m_{\mathrm{s}}$ is equal to $20 \mathrm{ps}^{-1}$. We can observed $\mathrm{B}_{\mathrm{s}}^{0}$ oscillation with a significance of at least $5 \sigma$ up to $68 \mathrm{ps}^{-1}$. Hence $\Delta m_{\mathrm{s}}$ range will be explored well beyond the Standard Model prediction.

The $\mathrm{B}_{\mathrm{s}}^{0}$ mixing phase, $\phi_{s}$, will be measured using the CP asymmetry in $\mathrm{B}_{\mathrm{s}}^{0} \rightarrow \mathrm{J} / \psi \phi$ events. This channel is the $\mathrm{B}_{\mathrm{s}}^{0}$ counterpart of the golden mode $\mathrm{B}^{0} \rightarrow \mathrm{J} / \psi \mathrm{K}_{\mathrm{S}}^{0}$. The $\mathrm{J} / \psi$ is reconstructed in $\mu^{+} \mu^{-}$or $\mathrm{e}^{+} \mathrm{e}^{-}$and the $\phi$ in $K^{+} K^{-}$. An angular analysis of the decays products is necessary to separate the CP-even from the CP-odd contributions. The $\phi_{s}$ phase can also be extracted from pure $\mathrm{CP}$ eigenstates like $\mathrm{B}_{\mathrm{s}}^{0} \rightarrow \mathrm{J} / \psi \eta$ or $\mathrm{B}_{\mathrm{s}}^{0} \rightarrow \eta_{\mathrm{c}} \phi$. These channels have a much lower statistics, but do not require an angular analysis. Combining all these modes, we expected a statistical uncertainty at the level of 0.013 radians with $10 \mathrm{fb}^{-1}$. The Standard Model predicts $\phi_{s}$ around -0.036 , but values order of magnitude higher can be expected in the presence of new physics.

\section{The $\alpha$ phase}

The Snyder and Quinn prescription ${ }^{6}$ to measure $\alpha$ has been studied in LHCb. The decays of $\mathrm{B}^{0}$ meson to $\pi^{+} \pi^{-} \pi^{0}$ is exploited, assuming that the intermediate $\rho \pi$ state is dominant. Compared to two-body decays approach, discrete ambiguities are theorically removed and an independent measurement of tree and penguin parameters is possible. The

$\overline{{ }^{\mathrm{a}} \text { In nominal condition, we expected }} 2 \mathrm{fb}^{-1}$ per year. 
$\mathrm{B}^{0} \rightarrow \pi^{+} \pi^{-} \pi^{0}$ selection is based on a multivariate analysis. Both $\pi^{0}$ giving merged and resolved clusters in the electromagnetic calorimeter are exploited. We expect 14,000 events per year after trigger, reconstruction and offline selection. The background-over-signal ratio from inclusive $b \bar{b}$ events has been estimated to be less than 1. The full Monte Carlo parameters are used to build toy Monte Carlo experiments, in order to assess the sensitivity to $\alpha$. Eleven-parameter likelihood fits are performed in time-dependent Dalitz space, on hundreds of toy Monte Carlo experiments. Assuming a flat and resonant background, we conclude that a statistical uncertainty of 10 degrees on $\alpha$ can be reached with $2 \mathrm{fb}^{-1}$.

\section{The $\gamma$ phase}

The $\gamma$ phase can be measured with at least six methods and only two are discussed here. The first one uses $\mathrm{B}_{\mathrm{s}}^{0} \rightarrow \mathrm{D}_{\mathrm{s}}^{\mp} \mathrm{K}^{ \pm}$events. Inteference between two tree diagrams and $\mathrm{B}_{\mathrm{s}}^{0}$ mixing in $\mathrm{B}_{\mathrm{s}}^{0} \rightarrow \mathrm{D}_{\mathrm{s}}^{\mp} \mathrm{K}^{ \pm}$allows an extraction of $\gamma+\phi_{\mathrm{s}}$ from two time-dependent decay rate asymmetries. The mistag fraction can be extracted from the $\mathrm{B}_{\mathrm{s}}^{0} \rightarrow \mathrm{D}_{\mathrm{s}}^{-} \pi^{+}$sample and $\phi_{\mathrm{s}}$ is measured with $\mathrm{B}_{\mathrm{s}}^{0} \rightarrow \mathrm{J} / \psi \phi$, as explained previously. Sensitivity have been estimated using fast Monte Carlo programs. The uncertainty on $\gamma$ is 14 degrees after one year of data taking. This assumes $\Delta m_{\mathrm{s}}=20 \mathrm{ps}^{-1}, \Delta \Gamma_{\mathrm{s}} / \Gamma_{\mathrm{s}}=0.1, \gamma$ between 55 and 105 degrees and the strong phases difference between the 2 tree diagrams to be below 20 degrees. This method has no theoretical uncertainties and is insensitive to new physics in the $\mathrm{B}_{\mathrm{s}}^{0}$ mixing.

The ADS method ${ }^{7}$ exploits the interference between the favoured and doubly Cabibbo-suppressed decay modes of neutral D mesons to states such as $\mathrm{K} \pi$ in charged B decays to DK. For these decays, the reversed suppression of the $\mathrm{D}$ decays relative to the $\mathrm{B}$ decays results in similar amplitude and big interference effects. No tagging nor proper time measurement is necessary, hence it is just a counting experiment. The interference depends on five parameters: $\gamma, r_{B}$, the ratio between the colour-allowed and colour-suppressed $\mathrm{B}$ decays, $\delta_{B}$, the strong phase difference between the two diagrams, $r_{D}^{K \pi}$, the ratio in magnitude of the two $\mathrm{D}$ decay processes and $\delta_{D}^{K \pi}$, the strong phase difference between the two $\mathrm{D}$ diagrams.

Four possible $\mathrm{B}$ decays have to be reconstructed: $\mathrm{B}^{-} \rightarrow\left(\mathrm{K}^{-} \pi^{+}\right)_{\mathrm{D}} \mathrm{K}^{-}$, $\mathrm{B}^{-} \rightarrow\left(\mathrm{K}^{+} \pi^{-}\right)_{\mathrm{D}} \mathrm{K}^{-}, \mathrm{B}^{+} \rightarrow\left(\mathrm{K}^{+} \pi^{-}\right)_{\mathrm{D}} \mathrm{K}^{+}, \mathrm{B}^{+} \rightarrow\left(\mathrm{K}^{-} \pi^{+}\right)_{\mathrm{D}} \mathrm{K}^{+}$. The first and the third decays are favoured. The second and the last are suppressed, but have interference term appearing at leading order, so that they are particularly sensitive to $\delta_{D}^{K \pi}$ and $\gamma$. Even though they are suppressed, 
the expected statistics in 1 year is 50 times higher that what is currently available at the $\mathrm{B}$ factories. Measuring the relative rates of these 4 processes give three observables which depends on four unknowns: $r_{B}, \gamma, \delta_{B}$ and $\delta_{D}^{K \pi}$. In order to constrain the problem, it is necessary to look for the $\mathrm{D}$ decay into another final state such as $\mathrm{D} \rightarrow \mathrm{K} \pi \pi \pi$.

Toy Monte Carlo experiment have been performed to estimate the sensitivity to $\gamma$. The level of background has been estimated to be around 0.5 for the two-body D decays, but is not yet known for the four-body D decays. Nevertheless, an arbitrarily $B / S$ has been introduced in the toy Monte Carlo and the result does not depend a lot on this $B / S$. The expected statistical uncertainty on $\gamma$ is around 5 degrees with $2 \mathrm{fb}^{-1}$.

\section{Rare decays}

The forward-backward asymmetry in the $\mu^{+} \mu^{-}$rest frame for $\mathrm{B}^{0} \rightarrow$ $\mu^{+} \mu^{-} \mathrm{K}^{* 0}$ decays is interesting because it allows to distinguish various extensions of the Standard Model ${ }^{8}$. The branching ratio of this decay is around $10^{-6}$. We expect to select $4,400 \mathrm{~B}^{0} \rightarrow \mu^{+} \mu^{-} \mathrm{K}^{* 0}$ events per year, with a $B / S$ ratio smaller than 2.6 . With $10 \mathrm{fb}^{-1}$, we expect to locate the zero of the forward backward asymetry to $\pm 0.53 \mathrm{GeV}$, which will allow to determine the ratio of the effective Wilson coefficients $C_{7}^{\mathrm{eff}}$ and $C_{9}^{\mathrm{eff}}$ with a $13 \%$ error.

\section{Conclusion}

LHCb will collect unprecedented statistics of $\mathrm{B}$ decays. $\mathrm{B}_{\mathrm{s}}^{0} \overline{\mathrm{B}}_{\mathrm{s}}^{0}$ oscillation will be measured with a $5 \sigma$ significance up to $68 \mathrm{ps}^{-1}$. Many measurements of rare decays and CP asymmetries will be performed. The statistical uncertainty on $\alpha, \gamma$ and $\phi_{s}$ after one year of data taking is expected to be 10,5 and 2 degrees, respectively. $\mathrm{CP}$ phases will be determined via channels with different sensitivity to new physics, so that detailed tests of the CKM description of the quark sector will be possible. A summary of the performance is given in Table 1 .

LHCb offers an excellent opportunity to spot new physics signal beyond Standard Model and to pin down its nature.

\section{Acknowledgments}

I would like to thanks the organizers of the 2006 Lake Louise Winter Institute and my LHCb colleagues for helping in preparing this talk. 
Table 1. Expected statistical performance of the LHCb experiment for key measurements with $2 \mathrm{fb}^{-1}$ (1 year of data taking). Untagged signal yields and background-over-signal ratios estimated from inclusive $\mathrm{b} \overline{\mathrm{b}}$ events are given.

\begin{tabular}{|c|c|c|c|c|}
\hline & Decay channel & Annual yield & $B / S$ & Stat. uncertainty \\
\hline$\gamma$ & $\begin{array}{l}\mathrm{B}_{\mathrm{s}}^{0} \rightarrow \mathrm{D}_{\mathrm{s}}^{\mp} \mathrm{K}^{ \pm} \\
\mathrm{B}^{0} \rightarrow \pi^{+} \pi^{-} \\
\mathrm{B}_{\mathrm{s}}^{0} \rightarrow \mathrm{K}^{+} \mathrm{K}^{-} \\
\mathrm{B}^{0} \rightarrow \mathrm{D}^{0}\left(\mathrm{~K}^{-} \pi^{+}\right) \mathrm{K}^{* 0} \\
\mathrm{~B}^{0} \rightarrow \overline{\mathrm{D}}^{0}\left(\mathrm{~K}^{+} \pi^{-}\right) \mathrm{K}^{* 0} \\
\mathrm{~B}^{0} \rightarrow \mathrm{D}_{\mathrm{CP}}^{0}\left(\mathrm{~K}^{+} \mathrm{K}^{-}\right) \mathrm{K}^{* 0} \\
\mathrm{~B}^{+} \rightarrow \mathrm{D}^{0}\left(\mathrm{~K}^{-} \pi^{+}\right) \mathrm{K}^{-} \\
\mathrm{B}^{+} \rightarrow \mathrm{D}^{0}\left(\mathrm{~K}^{+} \pi^{-}\right) \mathrm{K}^{-}\end{array}$ & $\begin{array}{c}5.4 \mathrm{k} \\
26 \mathrm{k} \\
37 \mathrm{k} \\
0.5 \mathrm{k} \\
2.5 \mathrm{k} \\
0.6 \mathrm{k} \\
60 \mathrm{k} \\
2 \mathrm{k}\end{array}$ & $\begin{array}{c}<<1 \\
<0.7 \\
0.3 \\
<0.3 \\
<2 \\
0.3 \\
0.5 \\
0.5\end{array}$ & $\begin{array}{l}\sigma(\gamma) \simeq 14^{o} \\
\sigma(\gamma) \simeq 6^{o} \\
\sigma(\gamma) \simeq 8^{o} \\
\sigma(\gamma) \simeq 5^{o}\end{array}$ \\
\hline$\alpha$ & $\mathrm{B}^{0} \rightarrow \pi^{+} \pi^{-} \pi^{0}$ & $14 \mathrm{k}$ & 0.8 & $\sigma(\alpha) \simeq 10^{\circ}$ \\
\hline$\phi_{\mathrm{s}}$ & $\begin{array}{l}\mathrm{B}_{\mathrm{s}}^{0} \rightarrow \mathrm{J} / \psi \phi \\
\mathrm{B}_{\mathrm{s}}^{0} \rightarrow \mathrm{J} / \psi \eta \\
\mathrm{B}_{\mathrm{s}}^{0} \rightarrow \eta_{\mathrm{c}} \phi\end{array}$ & $\begin{array}{c}125 \mathrm{k} \\
12 \mathrm{k} \\
3 \mathrm{k}\end{array}$ & $\begin{array}{l}0.3 \\
2-3 \\
0.7\end{array}$ & $\sigma\left(\phi_{\mathrm{s}}\right) \simeq 2^{o}$ \\
\hline$\Delta m_{\mathrm{s}}$ & $\mathrm{B}_{\mathrm{s}}^{0} \rightarrow \mathrm{D}_{\mathrm{s}}^{-} \pi^{+}$ & $80 \mathrm{k}$ & 0.3 & $\Delta m_{\mathrm{s}}$ up to $68 \mathrm{ps}^{-1}$ \\
\hline$\beta$ & $\mathrm{B}^{0} \rightarrow \mathrm{J} / \psi \mathrm{K}_{\mathrm{S}}^{0}$ & $216 \mathrm{k}$ & 0.8 & $\sin (2 \beta) \simeq 0.022$ \\
\hline $\begin{array}{l}\text { rare } \\
\text { decays }\end{array}$ & $\begin{array}{l}\mathrm{B}^{0} \rightarrow \mu^{+} \mu^{-} \mathrm{K}^{* 0} \\
\mathrm{~B}_{\mathrm{s}}^{0} \rightarrow \mu^{+} \mu^{-} \\
\mathrm{B}^{0} \rightarrow \mathrm{K}^{* 0} \gamma\end{array}$ & $\begin{array}{c}4.4 \mathrm{k} \\
17 \\
35 \mathrm{k} \\
\end{array}$ & $\begin{array}{l}<2.6 \\
<5.7 \\
<0.7\end{array}$ & $\begin{array}{c}\text { NP search } \\
\text { NP search } \\
\sigma\left(A_{C P}^{\text {dir }}\right) \simeq 0.01\end{array}$ \\
\hline
\end{tabular}

\section{References}

1. J. Nardulli, "LHCb Tracking System and Its Performance", Lake Louise Winter Institute 2006 in these proceedings.

2. P. Szczypka, "LHCb Particule Identification System and Its Performance", Lake Louise Winter Institute 2006 in these proceedings.

3. LHCb Collaboration, "LHCb Reoptimized Detector and Performance TDR" , CERN/LHCC 2003-30, LHCb TDR 9, 9 Sep 2003.

4. T. Maskawa and M. Kobayashi, "CP-Violation in the Renormalizable Theory of Weak Interaction", Prog. Th. Phys. 49 (1973) 652.

5. N. Cabibbo, "Unitarity Symmetry and Leptonic Decays", Phys. Rev. Lett. 10 (1963) 531.

6. A. Snyder and H. Quinn, Phys. Rev. D48, 2139 (1993).

7. D. Atwood, I. Dunietz and A. Soni Phys. Rev. Lett. 78, 3257 (1997).

8. P. Ball et al., "B decays at LHC",CERN-TH 2000-101, hep-ph/0003238 (2000). 